\title{
Predictors of 1-year compliance with adaptive servoventilation in patients with heart failure and sleep disordered breathing: preliminary data from the ADVENT-HF trial
}

To the Editor:

Despite its effectiveness in suppressing sleep disordered breathing (SDB), positive airway pressure therapy (PAP) is not always well tolerated by patients and long-term adherence can be problematic. Recently, two multicentre, randomised clinical trials (RCTs) tested the effects of PAP for patients with cardiovascular disease and co-existing SDB on morbidity and mortality with negative outcomes [1,2]. Relatively poor adherence to PAP therapy (mean 3.7 and $3.3 \mathrm{~h} \cdot$ day $^{-1}$, respectively) in these two trials might have contributed to their poor results. Indeed, higher PAP use per day is associated with better clinical outcomes than lower use [3].

The Effect of Adaptive Servo-ventilation on Survival and Cardiovascular Hospital Admissions in Patients with Heart Failure and Sleep Apnoea (ADVENT-HF) trial is a multinational RCT assessing the effects of treating SDB with peak flow targeted adaptive servoventilation (ASVpf) on morbidity and mortality in patients with heart failure and reduced ejection fraction (HFrEF) [4]. The purpose of this study was to determine the short- and long-term predictors of ASVpf adherence in an inception cohort of the ADVENT-HF trial. The trial has been registered (www.clinicaltrials.gov; NCT01128816), and its design, inclusion and exclusion criteria, and methods have been previously described [4].

The analysis included all patients from 38 sites randomised into the ASVpf treatment arm of the ADVENT-HF study before March 31, 2016, and for whom ASVpf adherence data were available at 1 and 12 months post-randomisation.

ASVpf compliance, defined as the average hours of use per day, was calculated for all patients and separately for the obstructive (OSA) and central sleep apnoea (CSA) groups. At 1 and 12 months, the percentage of patients compliant with ASVpf treatment, defined as use of $>50 \%$ of the baseline total sleep time per day [4], and the percentage with good compliance, defined as use of $\geqslant 4 \mathrm{~h} \cdot$ day $^{-1}$ were assessed. For patients who withdrew from the study or who discontinued using the device, hours of use were evaluated over the time during which the device was used and were imputed to be $0 \mathrm{~h}$ per night after discontinuation.

To define predictors of daily 1-month ASVpf use, univariable logistic regression was performed with the following as independent variables: age, body mass index, sex, presence of coronary artery disease, atrial fibrillation or flutter, country of site, baseline values of apnoea-hypopnoea index (AHI), Epworth Sleepiness Scale (ESS) score, sleep efficiency, arousal index, New York Heart Association class, N-terminal pro-B-type natriuretic peptide, left ventricular ejection fraction, Minnesota Living With Heart Failure Questionnaire scores, type of mask used, and leak at 1 month post-randomisation. A similar analysis was performed for daily 12-month ASVpf with the addition of the independent variables, daily hours of ASVpf use at 1 month, as well as AHI, ESS, type of mask and leak at 12 months post-randomisation. To define predictors of daily 12-month ASVpf use, a stepwise multiple regression analysis was performed.

@ERSpublications

Adherence to sleep apnoea treatment is essential to evaluate trials involving cardiovascular end-points. In patients with heart failure and obstructive or central sleep apnoea in the ADVENT-HF trial, adherence to adaptive servoventilation was very good. http://ow.ly/5sNz30mDj2W

Cite this article as: Perger E, Lyons OD, Inami T, et al. Predictors of 1-year compliance with adaptive servoventilation in patients with heart failure and sleep disordered breathing: preliminary data from the ADVENT-HF trial. Eur Respir J 2019; 53: 1801626 [https://doi.org/10.1183/13993003.01626-2018]. 
Of 177 subjects randomised to ASVpf, compliance data were available in 136 (91 with OSA and 45 with CSA). Of the remainder, 27 did not attend the scheduled clinic visit and 14 either refused to participate or withdrew consent after randomisation. Characteristics of those with OSA and CSA were similar, except for a higher AHI among CSA patients (median (interquartile range) 37.4 (29.7) versus 50.8 (24.2) events per $\mathrm{h}, \mathrm{p}=0.050)$.

ASVpf was effective in controlling both OSA and CSA. In those with predominantly OSA, while on ASVpf, the AHI decreased from mean \pm SD $42.7 \pm 19.8$ at baseline to $3.9 \pm 2.7$ events per $\mathrm{h} 1$ month later and to $3.3 \pm 2.8$ events per h 12 months later ( $\mathrm{p}<0.01$ for both). In those with predominantly CSA, while on ASVpf, the AHI decreased from $50.4 \pm 16.7$ at baseline to $5.3 \pm 3.7$ events per $\mathrm{h} 1$ month later and to $3.9 \pm 2.5$ events per $\mathrm{h}$ 12 months later ( $\mathrm{p}<0.01$ for both). This compares to a residual AHI of 6.6 events per h (mainly obstructive) 12 months post-randomisation on minute ventilation targeted adaptive servoventilation (ASVmv) in the SERVE-HF trial.

At 1 month, a nasal mask was used by 93 (68\%) of patients: 60 (66\%) with OSA and 33 (73\%) with CSA. At 12 months, the use of a nasal mask did not change: 86 (63\%) of patients, 57 (63\%) with OSA and 29 (64\%) with CSA. The remainder used a full-face mask.

By 1 month, of the 91 patients with OSA, only three (3\%) had discontinued ASVpf, while among the 45 with CSA, only one (2\%) patient had discontinued it. At 12 months, they increased to 18 (20\%) and five (11\%), respectively, or $23(17 \%)$ for all patients. The percentage of patients compliant [4] at 1 month was $86 \%$ among OSA and $87 \%$ among CSA patients, and 12 months it was $67 \%$ and $80 \%$, respectively. The median (interquartile range) daily use for all 136 subjects at 1 month was 4.7 (3.2) $\mathrm{h}$ and declined to $4.4(4.3) \mathrm{h}$ at 12 months $(\mathrm{p}<0.01)$. As shown in figure 1, daily ASVpf use declined from 4.6 to $4.1 \mathrm{~h} \cdot \mathrm{day}^{-1}$ in the OSA group, but remained stable at $5.2 \mathrm{~h} \cdot \mathrm{day}^{-1}$ in the CSA group.

Among patients with OSA, 56 (62\%) and 48 (53\%) used ASVpf $\geqslant 4$ h.day ${ }^{-1}$ at 1 and 12 months, respectively, while among patients with CSA, there were $30(67 \%)$ and $27(60 \%)$ at 1 and 12 months. No variable was a significant predictor of good compliance at 1 month. Hours of ASVpf use at 1 month was the only independent predictor of good ASVpf compliance at 12 months in the multivariate analysis of all patients (OR 2.02, 95\% CI 1.58-2.60 ( $\mathrm{p}<0.01)$ ) and in the subgroups separately (for OSA: OR 1.94, 95\% CI 1.27-2.78 ( $\mathrm{p}<0.01)$; for CSA; OR 2.12, 95\% CI 1.50-2.98 $(\mathrm{p}<0.01))$.

Both short- and long-term ASVpf compliance were good in these patients with HFrEF and SDB enrolled in the ADVENT-HF trial.

The Adaptive Servo-Ventilation for Central Sleep Apnea in Systolic Heart Failure (SERVE-HF) trial showed that ASVmv had no effect on the primary end-point, possibly due, in part, to low ASVmv compliance of $3.4 \mathrm{~h} \cdot \mathrm{day}^{-1}$ at $12 \mathrm{months}$ and $3.7 \mathrm{~h} \cdot \mathrm{day}^{-1}$ over the entire 5-year trial period [5, 6]. Only $48 \%$ of patients used ASVmv $\geqslant 4 \mathrm{~h} \cdot$ day $^{-1}$ over the trial period [2]. By contrast, ASVpf use in patients with CSA in the present study was higher in terms of hours of daily use and the proportion using it $\geqslant 4 \mathrm{~h} \cdot \mathrm{day}^{-1}$

FIGURE 1 Daily hours of peak flow targeted adaptive servoventilation (ASVpf) use at 1 and 12 months among obstructive (OSA) and central sleep apnoea (CSA) groups. Data are presented as median (interquartile range). Hours of use remained unchanged from 1 to 12 months in the CSA group at $5.2 \mathrm{~h}$, but hours of use declined significantly by $0.5 \mathrm{~h}$ from 1 to 12 months in the OSA group.

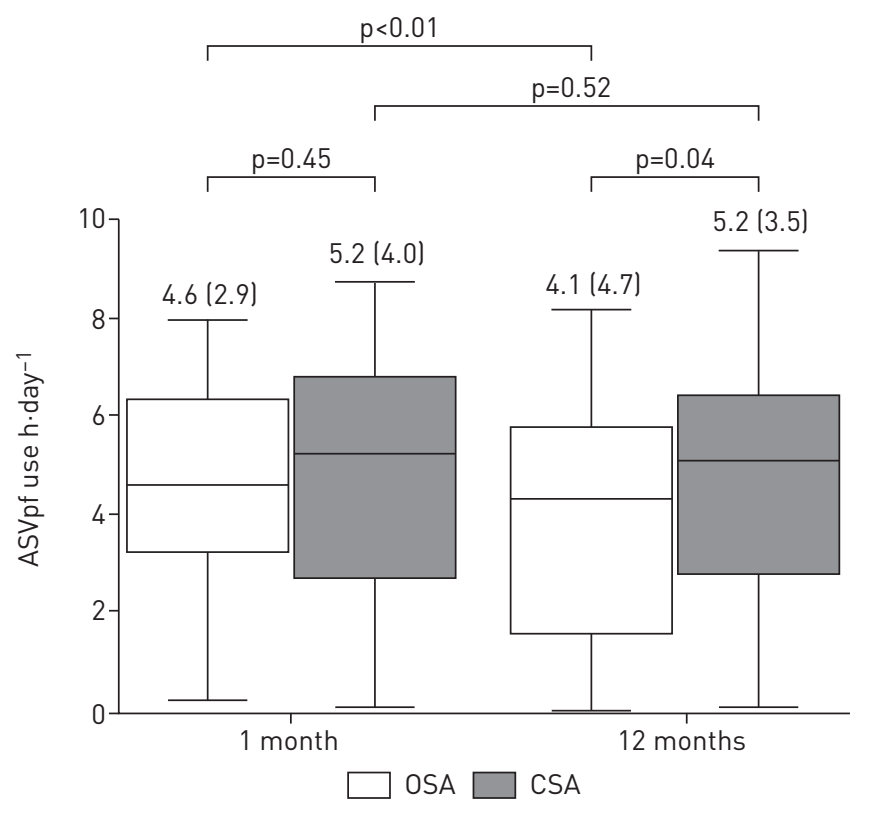


after 12 months, although the proportion of patients who discontinued ASVpf increased from $2 \%$ at 1 month to $11 \%$ at 12 months. Factors that might have contributed to better ASVpf compliance in the ADVENT-HF study include the technical characteristics of the ASVpf versus the ASVmv device used in SERVE-HF. ASVmv used relatively high default pressures with a minimum end-expiratory positive airway pressure (EPAP) of $5 \mathrm{cmH}_{2} \mathrm{O}$ and minimum inspiratory pressure support of $3 \mathrm{cmH}_{2} \mathrm{O}$, whereas ASVpf in the present study used lower default pressures with a minimum EPAP of $4 \mathrm{cmH}_{2} \mathrm{O}$ and a minimum pressure support of $0 \mathrm{cmH}_{2} \mathrm{O}$. These lower pressures might be better tolerated. In addition, unlike ASVmv that did not titrate EPAP to control obstructive events, ASVpf automatically titrates EPAP to control such events. This probably accounts for the lower residual AHI in patients on ASVpf, whether OSA or CSA, in the ADVENT-HF than in the SERVE-HF trial, where residual events were mainly obstructive. Centralised versus local interpretation of ASVpf titrations and prescription of ASVpf settings in ADVENT-HF versus SERVE-HF as well as more frequent interaction with patients due to semiannual versus annual follow-up visits in ADVENT-HF versus SERVE-HF may also have contributed to better ASVpf compliance. ASVpf compliance was also better in ADVENT-HF than ASVmv compliance in another recent RCT, in which daily use at 6 months was only $2.7 \mathrm{~h}[7]$.

Daily ASVpf use among patients with OSA in ADVENT-HF was also higher than continuous positive airway pressure (CPAP) use in the Sleep Apnea Cardiovascular Endpoints (SAVE) trial $\left(3.5 \mathrm{~h} \cdot \mathrm{day}^{-1}\right.$ 12 months post-randomisation) [1]. However, in contrast to patients with CSA, daily hours of ASVpf use among those with OSA declined at 12 months, in association with an increase in patients discontinuing ASVpf. The reason for the reduction in use over time was not clear. Once the trial is complete, it will be possible to assess outcomes data to determine if any of them are related to ASVpf compliance.

Recent RCTs testing CPAP therapy in nonsleepy OSA patients have demonstrated an absence of any effect on cardiovascular endpoints $[1,8,9]$. However, use of CPAP for $>4 \mathrm{~h} \cdot$ day $^{-1}$ was associated with a reduction in the incidence of the primary end-point $[8,9]$. Thus, there is a general tendency for PAP use of $\geqslant 4 \mathrm{~h} \cdot$ day $^{-1}$ to be associated with better outcomes. ASVpf has been shown to be more effective in suppressing OSA and CSA in patients with $\mathrm{HFrEF}$ than CPAP $[5,10,11]$.

The only factor that independently predicted daily hours of good ASVpf compliance 12 months post-randomisation was hours of ASVpf use at 1 month. These findings are compatible with previous evidence, indicating that patients' initial experience with the treatment may influence their long-term compliance $[12,13]$.

Whether better adherence to ASVpf therapy in the present study than in previous studies of patients with cardiovascular diseases and SDB $[1,2,7,8]$ will be associated with better clinical outcomes will not be known until completion of the trial.

Elisa Perger ${ }^{1,2}$, Owen D. Lyons ${ }^{1,2,3}$, Toru Inami ${ }^{1,2}$, Stephanie Smith ${ }^{1}$, John S. Floras ${ }^{4}$, Alexander G. $\operatorname{Logan}^{4}$ and $^{2}$ T. Douglas Bradley ${ }^{1,2,4}$ for the ADVENT-HF Investigators ${ }^{5}$

${ }^{1}$ Sleep Research Laboratory of the University Health Network Toronto Rehabilitation Institute, Toronto, ON, Canada. ${ }^{2}$ Centre for Sleep Medicine and Circadian Biology, Toronto, ON, Canada. ${ }^{3}$ Dept of Medicine Women's College Hospital, Toronto, ON, Canada. ${ }^{4}$ The University Health Network and Sinai Health System Dept of Medicine, Toronto, ON, Canada. ${ }^{5} \mathrm{~A}$ complete list of trial personnel, trial sites and investigators can be found at the end of this article.

Correspondence: T. Douglas Bradley, University Health Network Toronto General Hospital, 9N-943, 200 Elizabeth Street, Toronto, ON, M5G 2C4, Canada. E-mail: douglas.bradley@utoronto.ca

Received: Aug 262018 | Accepted after revision: Oct 292018

The trial is registered with Current Controlled Trials (www.controlled-trials.com; ISRCTN67500535) and Clinical Trials (www.clinicaltrials.gov; NCT01128816). The sponsoring institution, University Health Network, had formal data sharing agreements with all trial sites.

ADVENT-HF Trial Executive Committee: T.D. Bradley (Chair; Sleep Research Laboratory of the University Health Network Toronto Rehabilitation Institute, Centre for Sleep Medicine and Circadian Biology, and The University Health Network and Sinai Health System Dept of Medicine, Toronto, ON, Canada); J.S. Floras (Vice Chair; The University Health Network and Sinai Health System Dept of Medicine, Toronto, ON, Canada); A.G. Logan (Vice Chair; The University Health Network and Sinai Health System Dept of Medicine, Toronto, ON, Canada).

ADVENT-HF Steering Committee: T.D. Bradley (Chair); J.S. Floras (Member); A.G. Logan (Member); D.H. Delgado (Member; The University Health Network and Sinai Health System Dept of Medicine, Toronto, ON, Canada); R.J. Kimoff (Member; McGill University Health Center, Montreal, QC, Canada); M. Dunlap (Member; MetroHealth Medical Center, Cleveland, OH); G. Lorenzi-Filho (Member; Instituto do Coração do Hospital das Clínicas da FMUSP, Sao Paulo, Brazil); S. Redolfi (Member; AP-HP, Groupe Hospitalier Pitié-Salpêtrière Charles Foix, Paris, France); G. Parati (Member; Istituto Auxologico Italiano - Ospedale San Luca, Milan, Italy); M. Arzt (Member; Universitätsklinikum Regensburg, Regensburg, Germany); J. Durán Cantolla (Member; Hospital Universitario Txagorritxu, Vitoria, Spain); T. Kasai (Member; Juntendo University School of Medicine, Tokyo, Japan). 
ADVENT-HF Data and Safety Monitoring Committee: E.R. Smith (Chair; Cumming School of Medicine, University of Calgary, Calgary, AB, Canada); D. Johnstone (member; Dept of Medicine, University of Alberta, Edmonton, AB, Canada); R. Collins (member; Nuffield Dept of Population Health, University of Oxford, Oxford, UK).

ADVENT-HF Event Adjudication Committee: C. Joyner (Chair; Faculty of Medicine, University of Toronto and Sunnybrook Health Sciences Centre, Toronto, ON, Canada); J.S. Floras (Member; The University Health Network and Sinai Health System Dept of Medicine, Toronto, ON, Canada); S. Mak (Member; University Health Network and Sinai Health System Dept of Medicine, Toronto, ON, Canada); D.H. Delgado (Member; Faculty of Medicine, The University of Toronto and The University Health Network, Toronto, ON, Canada); A. Al-Hesayen (Member; Faculty of Medicine, The University of Toronto and St. Michael's Hospital, Toronto, ON, Canada); E. Oechslin (Member; Faculty of Medicine, The University of Toronto and The University Health Network, Toronto, ON, Canada); C. de Campos Gonzaga Carvalho (Member; Cardiac Prevention and Ambulatory Care Program, St. Michael's Hospital, Toronto, ON, Canada); E. Perger (Member; Sleep Research Laboratory of the University Health Network, Toronto Rehabilitation Institute, and Centre for Sleep Medicine and Circadian Biology, Toronto, ON, Canada); T. Inami (Member; Sleep Research Laboratory of the University Health Network, Toronto Rehabilitation Institute, and Centre for Sleep Medicine and Circadian Biology, Toronto, ON, Canada); K. Anderson (Member; Department of Medicine, Dalhousie University \& Nova Scotia Health Authority, Halifax, NS, Canada).

ADVENT-HF Investigators: T.D. Bradley, J.S. Floras, A.G. Logan, C.M. Ryan, D.H. Delgado, S. Mak and G. Tomlinson (University Health Network and Sinai Health System Dept of Medicine, Toronto, ON, Canada); R.S. Leung (St Michael's Hospital, Toronto, ON, Canada); D. Morrison (Capital District Health Authority, Halifax, NS, Canada); M. Fitzpatrick (Queen's University, Kingston, ON, Canada); P. Mayer (Hôpital Hôtel-Dieu du CHUM, Universite de Montreal, Montreal, QC, Canada); R.J. Kimoff (McGill University Health Center, Montreal, Canada); L. Mielniczuk and R. Beanlands (University of Ottawa Heart Institute, Ottawa, ON, Canada); F. Series (Institut Universitaire de Cardiologie et de Pneumologie de Québec, Québec, QC, Canada); J.A. Fleetham and N. Ayas (Vancouver General Hospital, University of British Columbia, Vancouver, BC, Canada); C. Demers (Hamilton General Hospital, Hamilton, ON, Canda); A.C.P. Powles (St Joseph's Healthcare, McMaster University, Hamilton, ON, Canada); C. Rinne (St Mary's General Hospital, Kitchener, ON, Canada); E. Giannouli (St Boniface General Hospital, Winnipeg, MB, Canada); M. Povitz (London Health Sciences Centre, Victoria Hospital, London, ON, Canda); A. Dal Nogare (Glacier View Research Institute, Kalispell, MT, USA); M. Dunlap (MetroHealth Medical Center, Cleveland, OH, USA); M. Shanmugasundaram (Southern Arizona VA Health Care System, Tucson, AZ, USA); G. Lorenzi-Filho and L. Drager (Instituto do Coração do Hospital das Clínicas da FMUSP, Sao Paulo, Brazil); L.R. Bittencourt (Centro de Desenvolvimento em Estudos Clínicos Brasil, Sao Paulo, Brazil); C. Amodeo and A. Bertolami (Instituto Dante Pazzanese de Cardiologia, Sao Paulo, Brazil); R. P. Pedrosa (Pronto Socorro Cardiologico de Pernambuco, Recife, Brazil); M. Mayos Pérez (Hospital de la Santa Creu i Sant Pau, Barcelona, Spain); P. Lloberes Canadell (Hospital Universitari Vall d’Hebron, Barcelona, Spain); J.F. Masa Jimenez (Hospital San Pedro de Alcántara, Cáceres, Spain); F. Barbé (Hospital Arnau de Vilanova, Lleida, Spain); N.P. González Mangado (Fundación Jiménez Diaz-CAPIO, Madrid, Spain); F. Del Campo (Hospital Universitario Rio Hortega, Valladolid, Spain); J. Durán Cantolla (Hospital Universitario Txagorritxu, Vitoria, Spain); J.M. Marin Trigo (Hospital Universitario Miguel Servet, Zaragoza, Spain); M. González Martinez (Hospital Universitario Marqués de Valdecilla, Santander, Spain); M. Arzt (Universitätsklinikum Regensburg, Regensburg, Germany); W. Randerath (Wissenschaftliches Institut Bethanien e.V., Solingen, Spain); M.T. La Rovere (Istituti Clinici Scientifici Maugeri (ICS Maugeri), Istituto Scientifico di Montescano, Pavia, Italy); G. Parati (Istituto Auxologico Italiano, Ospedale San Luca, Milan, Italy); C. Tantucci (Ospedale "Spedali Civili" Di Brescia, Brescia, Italy); A. Braghiroli (ICS Maugeri, Istituto Scientifico di Veruno, Veruno, Italy); D. Raccagni (U.O. Cardiologia, ASST Franciacorta, Chiari, Italy); S. Redolfi (AP-HP, Groupe Hospitalier Pitié-Salpêtrière Charles Foix, Paris, France); F. de Roquefeuil (AP-HP, Groupe Hospitalier Ambroise Paré, Boulogne-Billancourt, France); F. Goutorbe (Centre Hospitalier de Béziers, Béziers, France); P. Escourrou (AP-HP, Hôpital Antoine Béclère, Clamart, France); M-P. d’Ortho (AP-HP, Hôpital Bichat Claude Bernard, Paris, France); V. Puel (Nouvelle Clinique Bel Air, Bordeaux, France); R. Tamisier (Centre Hospitalier Universitaire de Grenoble, Grenoble, France); A. Williams (The Sleep Disorders Centre, Nuffield House, Guy's Hospital, London, UK); T. Kasai (Juntendo University School of Medicine, Tokyo, Japan); Y. Takata (Tokyo Medical University Hospital, Tokyo, Japan); K. Chin and K. Murase (Kyoto University Hospital, Kyoto, Japan); K. Narui and Y. Tomita (Toranomon Hospital, Tokyo, Japan).

Conflict of interest: E. Perger reports receiving grants from Philips Respironics during the conduct of the study. O.D. Lyons reports receiving grants from Philips Respironics during the conduct of the study. T. Inami reports receiving grants from Philips Respironics Japan during the conduct of the study. S. Smith reports receiving grants from Philips Respironics during the conduct of the study. J.S. Floras is Vice-Chair of the ADVENT-HF trial, the operation of which has been funded jointly by the Canadian Institutes of Health Research and Philips Respironics. A.G. Logan reports receiving grants from Philips Respironics during the conduct of the study. T.D. Bradley reports receiving grants from Philips Respironics during the conduct of the study.

Support statement: This study is jointly funded by a grant from the Canadian Institutes of Health Research (CIHR) and an unrestricted grant from Philips Respironics Inc. in accordance with CIHR's University Industry Partnership Program. E. Perger was supported by the University of Brescia (Brescia, Italy); O.D. Lyons by a joint Canadian Thoracic Society/ European Respiratory Society Peter Macklem Research Fellowship and the Joseph M. West Family Memorial Fund Postgraduate Research Award from the University of Toronto; T. Inami by unrestricted research fellowships from Nippon Medical School (Tokyo, Japan), Philips Respironics Japan and Fukuda Foundation for Medical Technology; J.S. Floras by a Canada Research Chair in Integrative Cardiovascular Biology; and T.D. Bradley by the Clifford Nordal Chair in Sleep Apnea and Rehabilitation Research and the Godfrey S. Pettit Chair in Respiratory Medicine.

\section{References}

1 McEvoy R, Antic N, Heeley E, et al. CPAP for prevention of cardiovascular events in obstructive sleep apnea. N Engl J Med 2016; 375: 919-931. 
2 Cowie MR, Woehrle $\mathrm{H}$, Wegscheider $\mathrm{K}$, et al. Adaptive servo-ventilation for central sleep apnea in systolic heart failure. N Engl J Med 2015; 373: 1095-1105.

3 Antic NA, Catcheside P, Buchan C, et al. The effect of CPAP in normalizing daytime sleepiness, quality of life, and neurocognitive function in patients with moderate to severe OSA. Sleep 2011; 22: 111-119.

4 Lyons OD, Floras JS, Logan AG, et al. Design of the effect of adaptive servo-ventilation on survival and cardiovascular hospital admissions in patients with heart failure and sleep apnoea: the ADVENT-HF trial. Eur J Heart Fail 2017; 19: 579-587.

5 Bradley TD, Floras JS. Adaptive servo-ventilation for central sleep apnea in heart failure. N Engl J Med 2016; 374: 689 .

6 Javaheri S, Brown LK, Randerath W, et al. SERVE-HF: more questions than answers. Chest 2016; 149: 900-904.

7 O'Connor CM, Whellan DJ, Fiuzat M, et al. Cardiovascular outcomes with minute ventilation-targeted adaptive servo-ventilation therapy in heart failure: the CAT-HF trial. J Am Coll Cardiol 2017; 69: 1577-1587.

8 Peker Y, Glantz H, Eulenburg C, et al. Effect of positive airway pressure on cardiovascular outcomes in coronary artery disease patients with non-sleepy obstructive sleep apnea: the RICCADSA randomized controlled trial. Am J Respir Crit Care Med 2016; 194: 613-620.

9 Barbe F, Duran-Cantolla J, Sanchez-de-la-Torre M, et al. Effect of continuous positive airway pressure on the incidence of hypertension and cardiovascular events in nonsleepy patients with obstructive sleep apnea: a randomized controlled trial. JAMA 2012; 307: 2161-2168.

10 Teschler H, Dohring J, Wang YM, et al. Adaptive pressure support servo-ventilation: a novel treatment for Cheyne-Stokes respiration in heart failure. Am J Respir Crit Care Med 2001; 164: 614-619.

11 Arzt M, Wensel R, Montalvan S, et al. Effects of dynamic bilevel positive airway pressure support on central sleep apnea in men with heart failure. Chest 2008; 134: 61-66.

12 Collen J, Lettieri C, Kelly W, et al. Clinical and polysomnographic predictors of short-term continuous positive airway pressure compliance. Chest 2009; 135: 704-709.

13 Budhiraja R, Parthasarathy S, Drake C, et al. Early CPAP use identifies subsequent adherence to CPAP therapy. Sleep 2007; 30: 320-324 